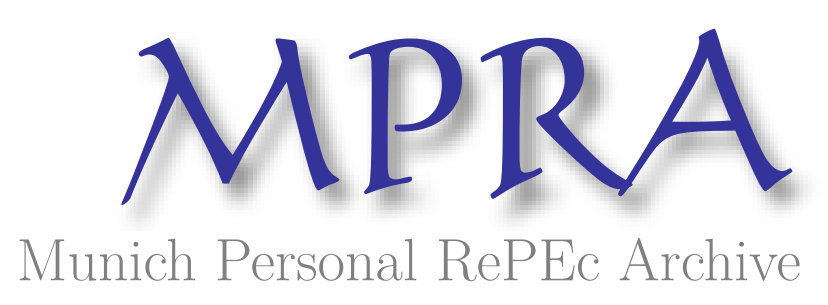

\title{
Bounded Interest Rate Feedback Rules in Continuous-Time
}

d'Albis, Hippolyte and Augeraud-Véron, Emmanuelle and Hupkes, Hermen Jan

7 March 2013

Online at https://mpra.ub.uni-muenchen.de/45424/

MPRA Paper No. 45424, posted 22 Mar 2013 15:21 UTC 


\title{
Bounded Interest Rate Feedback Rules in Continuous-Time ${ }^{1}$
}

\author{
Hippolyte d'Albis \\ Paris School of Economics, University Paris 1 \\ Emmanuelle Augeraud-Véron \\ MIA, University of La Rochelle \\ Hermen Jan Hupkes \\ University of Leiden
}

March 7, 2013

\footnotetext{
${ }^{1}$ We thank J.-P. Drugeon, G. Fabbri, E. Iliopoulos and A. Saidi for stimulating suggestions and comments. H. d'Albis acknowledges financial support from the European Research Council (ERC Stg Grant DU 283953). The usual disclaimer applies.
} 


\begin{abstract}
This paper analyses the dynamic consequences of interest rate feedback rules in a flexible-price model where money enters the utility function. Two alternative rules are considered based on past or predicted inflation rates. The main feature is to consider inflation rates that are selected over a bounded time horizon. We prove that if the Central Bank's forecast horizon is not too long, an active and forward-looking monetary policy is not destabilizing: the equilibrium trajectory is unique and monotonic. This is an advantage with respect to active and backward-looking policies that are shown to lead to a unique but fluctuating dynamic.
\end{abstract}

JEL Classification: E31, E43, E52.

Keywords: Interest Rate Rules, Indeterminacy, Functionnal Equations. 


\section{Introduction}

Since McCallum (1981), monetary feedback rules have been studied to reestablish determinacy in monetary models (Bernanke and Woodford, 1997, Woodford, 2003). The choices pertaining to the modeling of the variables' timing are particularly important in those models (Carlstrom and Fuerst, 2000, 2001), and recommendations may vary depending on whether a discreteor continuous-time representation is chosen (Dupor, 2001, Carlstrom and Fuerst, 2003, 2005). In this article, we study and extend a framework initially proposed by Benhabib (2004) that mix both representations.

This is a continuous-time model with flexible prices and money in the utility function where the current nominal interest rates are set by a Central Bank according to a feedback rule, as in Benhabib et al. (2001). The main feature is that interest rate policy is not defined according to the current value of inflation, nor on its value over an infinite horizon, but on its values over a finite horizon. With this assumption, the continuous-time model is more similar to traditional discrete-time models used in the literature. It turns out to be easier to solve. As recalled by Benhabib (2004), with discrete-time frameworks, the order of the difference equation that describes the equilibrium increases with the number of lagged inflation rates. For instance, the Taylor (1993) rule based on inflation recorded over the last four quarters implies that dynamics are described by an equation of at least 4th order, which is difficult to study analytically. In continuous-time, considering a bounded backward-looking rule leads to a dynamic that is described by a delay differential equation. We show how to use recent mathematical results on functional differential equations (d'Albis et al. 2012, 2013) to easily solve analytically the issue of the determinacy of the equilibrium in the framework 
developed by Benhabib (2004). We also extend his analysis by considering a forward-looking rule that leads to an advance differential equation. According to Clarida et al. (1998, 2001), forward looking rules seem to be more realistic while Orphanides (2001) show that they provide a better fit of real time data than current or backward-looking rules.

The results of our study, where consumption and money are assumed to be complementary in the utility function, are as follows. When the interest rate rule is a function of past inflation rates, equilibrium is indeterminate if the policy is passive and unique if the policy is active. This result holds whatever the length of the horizon for which inflation rates are taken into account in the interest rate rule. Moreover, the dynamics are characterized by short-term fluctuations, even when the equilibrium is determinate. When the interest rate rule is a function of future inflation rates, equilibrium remains indeterminate if the policy is passive; on the other hand, if the policy is active, equilibrium is unique provided that the Central Bank's forecast horizon is not too distant. This implies that fixing a short-term horizon may be an alternative to an aggressive interest rates policy. This complements earlier findings from models with sticky prices (Batini and Pearlman, 2002, Batini et al. 2004, 2006, Levine et al. 2007).

We recognize certain limitations to our study. First of all, we do not investigate the global dynamics of the system despite the fact that several studies have demonstrated its importance in interest rate policies (Benhabib et al. 2003, Eurepi, 2005, Cochrane, 2011). Similarly, we do not study permanent oscillations, especially those generated by Hopf bifurcations, despite the fact they may appear in this approach. In both instances, we are limited by the fact that there are no general theorems for the type of equations being considered. 
In Section 2, we present the model whose solution is studied in Sections 3 and 4, where we make the distinction between backward-looking and forwardlooking policies. Our conclusions are discussed in Section 5.

\section{Feedback rules over a bounded horizon}

We consider a model that is similar to those studied by Benhabib et al. (2001) and Benhabib (2004). This is a flexible-price model where nominal interest rates are set by the Central Bank as a function of past or forecasted inflation rates. The novelty is to consider that backward and forward horizons of the Central Bank are bounded.

Time is continuous and is denoted by $t \in \mathbb{R}_{+}$. Let $c(t), m(t)$ and $a(t)$ be respectively real consumption, real balances held for non-production purposes and real financial wealth. The household's problem is:

$$
\begin{array}{l|l}
\max _{\{c, m, a\}} \int_{0}^{\infty} e^{-r t} U(c(t), m(t)) d t \\
\text { s.t. } & \begin{array}{l}
a^{\prime}(t)=[R(t)-\pi(t)] a(t)-R(t) m(t)+Y-c(t)-\tau(t), \\
a(0)>0 \text { given, } \\
\lim _{t \rightarrow+\infty} a(t) e^{-\int_{0}^{t}[R(z)-\pi(z)] d z} \geq 0,
\end{array}
\end{array}
$$

where $r>0$ and $Y>0$ denote the rate of time preference and the output, respectively. $R(t), \pi(t)$ and $\tau(t)$ are perfectly anticipated by the household and denote the trajectories of the nominal interest rate, the inflation rate and the real lump-sum taxes. The instant utility function $U(c(t), m(t))$ is strictly increasing $\left(U_{c}>0, U_{m}>0\right)$ and strictly concave $\left(U_{c c}>0, U_{m m}>0\right)$ in both arguments. Moreover, consumption and real balances are assumed to be complementary $\left(U_{c m}>0\right)$, which implies that they are both normal goods. The cases where real balances are substitutable with consumption 
or are productive are immediate extensions of the present work. We assume that the fiscal policy is Ricardian.

Letting $\lambda(t)$ be the Lagrange multiplier associated with the household's instant budget constraint, the first order conditions are:

$$
\begin{aligned}
\lambda(t) & =U_{c}(c(t), m(t)), \\
R(t) & =\frac{U_{m}(c(t), m(t))}{U_{c}(c(t), m(t))}, \\
\lambda^{\prime}(t) & =\lambda(t)[r+\pi(t)-R(t)],
\end{aligned}
$$

together with the household's instant budget constraint given in problem (1) and the transversality condition:

$$
\lim _{t \rightarrow+\infty} a(t) e^{-\int_{0}^{t}[R(z)-\pi(z)] d z}=0
$$

We assume that nominal interest rates are set by the Central Bank according to the following rule:

$$
R(t)=\rho\left(\left(1-\chi^{b}-\chi^{f}\right) \pi(t)+\chi^{b} \pi^{b}(t)+\chi^{f} \pi^{f}(t)\right),
$$

where $\pi^{b}(t)$ and $\pi^{f}(t)$ denote backward and forward indicators of inflation defined respectively by the weighted averages of past and expected future rates of inflation. The indicators write:

$$
\pi^{b}(t)=\frac{\int_{t-\Omega^{b}}^{t} e^{\beta^{b}(u-t)} \pi(u) d u}{\int_{-\Omega^{b}}^{0} e^{\beta^{b} u} d u} \text { and } \pi^{f}(t)=\frac{\int_{t}^{t+\Omega^{f}} e^{-\beta^{f}(u-t)} \pi(u) d u}{\int_{0}^{\Omega^{f}} e^{-\beta^{f} u} d u}
$$

where $\Omega^{b}>0$ and $\Omega^{f}>0$ respectively denote the bounded backward and forward horizon of the Central Bank, where $\beta^{b}>0$ and $\beta^{f}>0$ are the weights associated to inflation rates within the indicators, and where $\chi^{b} \geq 0$ and $\chi^{f} \geq 0$, which satisfy $\chi^{b}+\chi^{f}<1$, are the weights given by the Central Bank to the backward and forward indicators. Below, we consider either a backward-looking feedback rule, for $\chi^{f}=0$, or a forward-looking one, for 
$\chi^{b}=0$. Finally, we assume that $\rho^{\prime}(x)>0$ for all $x \in \mathbb{R}_{+}$. As in Leeper (1991), the policy is considered to be active for $\rho^{\prime}>1$ and passive for $\rho^{\prime}<1$. The interest rate rule we consider is more general than the one of Benhabib et al. (2001) who study the limit cases $\Omega^{b} \rightarrow+\infty$ and $\Omega^{f} \rightarrow+\infty$. It is, moreover, similar to Benhabib (2004) who assumes $\chi^{f}=0, \chi^{b}=1$ and considers the following indicator:

$$
\pi^{b}(t)=\frac{\int_{-\infty}^{t-\Omega^{b}} e^{\beta^{b}(u-t)} \pi(u) d u}{\int_{-\infty}^{-\Omega^{b}} e^{\beta^{b} u} d u} .
$$

Benhabib (2004) hence assumes that information about past inflation rates is obtained by the Central Bank after a delay while we assume that, after some time, information conveyed by past inflation rate is not considered as relevant by the Central Bank. Formally, the advantage of (8) is that the functional equation may reduce to a difference equation, which makes the comparison with discrete time models easier.

In equilibrium, the goods market must clear, which writes: $c(t)=Y$. By replacing this equilibrium condition in (2) and (3), we obtain: $\lambda(t)=$ $U_{c}(Y, m(t))$, and $R(t)=\frac{U_{m}(Y, m(t))}{U_{c}(Y, m(t))}$. Let us use the latter to define the implicit function $m(t)=M(R(t))$ and replace it in the former. We differentiate with respect to time the new equation and rearrange using (4) to obtain:

$$
R^{\prime}(t)=\Lambda(R(t))[R(t)-r-\pi(t)]
$$

with

$$
\Lambda(R(t)) \equiv \frac{U_{m}(Y, M(R(t)))}{U_{c}(Y, M(R(t)))}-\frac{U_{m m}(Y, M(R(t)))}{U_{c m}(Y, M(R(t)))} .
$$

The dynamics of the variables $R(t), \pi(t), \pi^{b}(t), \pi^{f}(t)$ for all $t \in \mathbb{R}_{+}$are characterized by a system composed of two algebraic equations given in (7), a static equation (6), and a differential equation (9). We remark that the algebraic equations reduce to differential equations with a discrete delay or a 
discrete advance when differentiated once with respect to time. $R(t)$ and $\pi(t)$ are forward variables, with $R\left(0^{+}\right)$and $\pi\left(0^{+}\right)$that are not given. Initial conditions for these two variables hence write: $R(\theta)=\bar{R}(\theta) \in C\left(\left[-\Omega^{b}, 0\right), \mathbb{R}_{+}\right)$ given and $\pi(\theta)=\bar{\pi}(\theta) \in C\left(\left[-\Omega^{b}, 0\right), \mathbb{R}_{+}\right)$given. In principle, we also have that $\pi^{b}(\theta)=\bar{\pi}^{b}(\theta) \in C\left(\left[-\Omega^{b}, 0\right), \mathbb{R}_{+}\right), R\left(0^{-}\right)$given. However, using the first equation of (7) computed for $t=0$, we see that $\pi^{b}(t)$ is a backward variable whose initial condition is now: $\pi^{b}(\theta)=\bar{\pi}^{b}(\theta)$ given for $\theta \in\left[-\Omega^{b}, 0\right]$ and where $\pi^{b}\left(0^{+}\right)$is given by the algebraic equation. This implies that $\bar{\pi}^{b}(\theta)$ may be discontinuous at $\theta=0$. Conversely, using (6) computed at $t=0$, we see that $\pi^{f}(t)$ is a forward variable with the following initial condition: $\pi^{f}(\theta)=\bar{\pi}^{f}(\theta) \in C\left(\left[-\Omega^{b}, 0\right), \mathbb{R}_{+}\right)$given. $\pi^{f}\left(0^{+}\right)$will have to be determined and will be possibly different from $\pi^{f}\left(0^{-}\right)$.

For the perfect-foresight equilibrium we consider, it is implicitly assumed that the initial price level is given. More precisely, for all $\theta \in\left[-\Omega^{b}, 0\right)$, the price level, denoted $P(\theta)$, solves:

$$
P(\theta)=P\left(-\Omega^{b}\right) e^{\int_{-\Omega^{b}}^{0} \pi(u) d u} .
$$

Hence, this is $P\left(0^{+}\right)$(which is allowed to be different from $P\left(0^{-}\right)$) that is arbitrarily chosen in our framework. It is well known since Sargent and Wallace (1975) that the initial price level cannot be determined in this type of model.

As in Benhabib et al. (2001), we are going to study the trajectories for which the inflation rate converges to a constant. We provide a local analysis of these trajectories and, therefore, restrict ourselves to neighborhoods of a steady-state defined as a collection $\left(R_{*}, \pi_{*}, \pi_{*}^{b}, \pi_{*}^{f}\right)$ that solves $\pi_{*}=\pi_{*}^{b}=$ $\pi_{*}^{f}=R_{*}-r$, and

$$
\rho\left(\pi_{*}\right)-\pi_{*}-r=0
$$


We assume $^{1}$ there exists a steady-state that satisfies: $\rho^{\prime}\left(\pi_{*}\right) \neq\left(1-\chi^{b}-\chi^{f}\right)^{-1}$. It is also important (Benhabib et al. 2002 and Cochrane, 2011) to assume the uniqueness of the steady-state.

Since we consider functional equations, the Hartman-Grobman theorem does not apply to our problem, and we have to prove that studying the linearized system is not misleading. This is done by establishing the following result.

Lemma 1. In the neighborhood of the steady-state, the dynamics of the system of equations (6), (7) and (9) behave similarly to those of its linearized counterpart, provided that the latter is hyperbolic.

Proof. See Appendix.

The condition in Lemma 1 will be satisfied below. We now compare the dynamics induced by the choice of the Central Bank to follow either a backward-looking interest rate rule or a forward-looking one.

\section{Backward-looking feedback rules}

We consider the case where nominal interest rates are set by the Central Bank as a function of past and present inflation rates. For $\chi^{f}=0$, the

\footnotetext{
${ }^{1}$ Equivalently, we could have given straightforward conditions on the limits of function $\rho$ that would be sufficient for existence of a real solution to (12). Note also that since $\rho^{\prime}>0$, existence implies uniqueness.
} 
perfect-foresight equilibrium satisfies:

$$
\left\{\begin{array}{l}
R^{\prime}(t)=\Lambda(R(t))\left[R(t)-r-\frac{\rho^{-1}(R(t))-\chi^{b} \pi^{b}(t)}{\left(1-\chi^{b}\right)}\right] \\
\pi^{b}(t)=\frac{\int_{t-\Omega^{b}}^{t} e^{\beta^{b}(u-t)}\left[\rho^{-1}(R(u))-\chi^{b} \pi^{b}(u)\right] d u}{\left(1-\chi^{b}\right) \int_{-\Omega^{b}}^{0} e^{\beta^{b}} d u} \\
\pi^{b}(\theta)=\bar{\pi}^{b}(\theta) \text { given for } \theta \in\left[-\Omega^{b}, 0\right] \\
\lim _{t \rightarrow+\infty} \pi^{b}(t)=\pi_{*}, \lim _{t \rightarrow+\infty} R(t)=R_{*} .
\end{array}\right.
$$

The system (13) is defined as a two dimensional system composed by an ordinary differential equation and an algebraic equation with a continuum of delays, which reduces to a delay differential equation when differentiated once with respect to time. There is one forward variable, $R(t)$, and one backward variable, $\pi^{b}(t)$, for which a discontinuity is allowed at $t=0$, as $\pi^{b}\left(0^{+}\right)$is given by the algebraic equation.

We are going to study the local existence and uniqueness of solutions a such a system by applying Theorem 1 in d'Albis et al. (2013). Let us denote by $n^{+}$the number of roots with positive real parts of the characteristic equation associated with the linearized counterpart of system (13). We remark that if $n^{+}>0$, then $0<s \leq n^{+}$, where $s$ is the dimension of the unstable eigenspace.

Lemma 2. The characteristic equation associated with the linearized counterpart of system (13) has $n^{+}=0$ if $\rho^{\prime}\left(\pi_{*}\right) \in(0,1)$, and $n^{+}=1$ if $\rho^{\prime}\left(\pi_{*}\right)>1$

Proof. See Appendix.

By applying Theorem 1 in d'Albis et al. (2013), we conclude that the equilibrium is locally indeterminate if the monetary policy is passive while it is locally unique if the policy is active. In other words, if the policy is 
passive the steady-state is stable in the sense that there is a continuum of $R\left(0^{+}\right)$that initiate a converging trajectory. If the policy is active, there is a unique solution for $R\left(0^{+}\right)$and the steady-state is saddle-point stable. This confirms and extends the results obtained by Benhabib (2004) for the case where recent and contemporaneous inflation rates are not included in the rule. Benhabib found that following a passive policy is a sufficient condition for local indeterminacy whereas an active policy is a necessary condition for uniqueness. With Lemma 2, we show that an active policy is also a sufficient condition for local uniqueness.

To see the importance of the bounded backward horizon on this result, we now consider the case where $\Omega^{b} \rightarrow+\infty$, which is also studied in Benhabib (2004) who considers the limit case of no informational delay. The system (13) reduces to a system of two ordinary differential equations. The next Lemma studies the roots of the corresponding characteristic function.

Lemma 3. Assume that $\Omega^{b} \rightarrow+\infty$. The characteristic equation has $n^{+}=0$ if $\rho^{\prime}\left(\pi_{*}\right) \in(0,1)$, and $n^{+}=1$ if $\rho^{\prime}\left(\pi_{*}\right)>1$.

Proof. See Appendix.

We see that the determinacy property is the same as in Lemma 2: there are multiple equilibria if the policy is passive but the equilibrium is unique if the policy is active. The finite delay has, thus, no impact on the determinacy of the equilibrium. This result generalizes what Benhabib et al. (2001) showed about the determinacy condition being the same for currentlooking rules and backward-looking rules with an infinite horizon. However, the dynamics are qualitatively quite different. In the case where the policy is active, the dynamics converge with exponentially decreasing fluctuations toward the steady-state if $\Omega^{b}$ is finite whereas they are monotonic if $\Omega^{b}$ is 
infinite. This difference is due to the complex roots that generically emerge when using delay differential equations (see e.g. Boucekkine et al. 2004). As fluctuations in $R(t)$ leads to fluctuations in the instantaneous utility of the representative household, a welfare analysis would lead us to recommend an infinite horizon rule rather than a finite one.

There is an assumption that is important in our setting. We assume that contemporaneous inflation is necessarily included in the interest rate rule, which formally comes from assumption: $\chi^{b}<1$. Conversely, if it is assumed that $\chi^{b}=1$, the rule (6) becomes $R(t)=\rho\left(\pi^{b}(t)\right)$ and the problem is significantly modified as the nominal interest rate becomes a backward variable. Moreover, using (7) and (9), the dynamics reduce to a single equation that writes:

$$
R(t)=\rho\left(\frac{\int_{t-\Omega^{b}}^{t} e^{\beta^{b}(u-t)}\left[R(u)-\frac{R^{\prime}(u)}{\Lambda(R(u))}\right] d u}{\int_{-\Omega^{b}}^{0} e^{\beta^{b} u} d z}-r\right) .
$$

This is a delay differential equation of neutral type and theorems in d'Albis et al. (2013) do not cover this kind of equation ${ }^{2}$.

\section{Forward-looking feedback rules}

We now consider the case where nominal interest rates are set by the Central Bank as a function of expected and present inflation rates. For $\chi^{b}=0$, the perfect-foresight equilibrium satisfies:

$$
\left\{\begin{array}{l}
R^{\prime}(t)=\Lambda(R(t))\left[R(t)-r-\frac{\rho^{-1}(R(t))-\chi^{f} \pi^{f}(t)}{\left(1-\chi^{f}\right)}\right], \\
\pi^{f}(t)=\frac{\int_{t}^{t+\Omega^{f}} e^{-\beta^{f}(u-t)}\left[\rho^{-1}(R(u))-\chi^{f} \pi^{f}(u)\right] d u}{\left(1-\chi^{f}\right) \int_{0}^{\Omega f} e^{-\beta^{f} u} d u} \\
\lim _{t \rightarrow+\infty} \pi^{f}(t)=\pi_{*}, \lim _{t \rightarrow+\infty} R(t)=R_{*} .
\end{array}\right.
$$

\footnotetext{
${ }^{2}$ A possibility would be to use optimal control as in Boucekkine, Fabbri and Pintus (2012).
} 
System (15) is similar to system (13) except that the algebraic equation includes advances rather than delays. Moreover, the two variables, $R(t)$ and $\pi^{f}(t)$, are forward. To study the local existence and uniqueness of solutions of (15), Theorem 2 in d'Albis et al. (2013) can easily be applied to its linearized counterpart. Using Corollary 2, we conclude that there exists at least one solution to system (15). The next Lemma studies local uniqueness of the equilibrium. We denote by $n^{-}$the number of roots of the characteristic equation that have negative real parts.

Lemma 4. Let $\bar{\beta}^{f} \equiv \Lambda\left(R_{*}\right)$ and assume that $\beta^{f}>\bar{\beta}^{f}$. The characteristic equation associated with the linearized counterpart of system (15) has $n^{-}=1$ if $\rho^{\prime}\left(\pi_{*}\right) \in(0,1)$, and $n^{-}=0$ if $\rho^{\prime}\left(\pi_{*}\right)>1$.

Proof. See Appendix.

Lemma 4 states that if the weight associated to future inflation rates is sufficiently low the determinacy typology of the equilibrium is the same as the one obtained with backward-looking feedback rules. If $\beta^{f}$ is above a finite threshold, there exists a unique equilibrium if the policy is active (the steady-state is unstable and both $R(t)$ and $\pi^{f}(t)$ jump to their long-run values) while the equilibrium is locally indeterminate if the policy is passive. This result can be interpreted by a continuity argument by noticing that in the limit case where $\beta^{f} \rightarrow 0$, one gets a current-looking rule. Contrarily to backward-looking feedback rules, there are no exponentially decreasing fluctuations in the dynamics. Moreover, as $R(t)=R_{*}$ and $\pi^{f}(t)=\pi_{*}$ for all $t$, the welfare of the representative household does not depend on parameters $\left(\beta^{f}, \bar{\Omega}^{f}\right)$.

The threshold $\bar{\beta}^{f}$ constitutes a standard limit to the size of feedback on forward feedback. For low discount factors (i.e. for $\beta^{f}<\bar{\beta}^{f}$ ), the characteri- 
zation of the roots is tedious but other situations may arise. For instance, it can be shown that there exist sets of parameters such that the equilibrium is indeterminate whatever the value of $\rho^{\prime}\left(\pi_{*}\right)$ and others that exhibit pure imaginary roots. This confirms previous results on the destabilizing effects of forward-looking feedback rules (Woodford, 1999, and Benhabib et al. 2001 or Bernanke and Woodford, 1997, in a discrete time model).

Nevertheless, in our setting, a natural way to reduce the weight associated to future inflation rates can be obtained by modifying the upper bound of the integral, $\Omega^{f}$. This is studied in the next Lemma.

Lemma 5. There exists $\bar{\Omega}^{f}>0$ such that for $\Omega^{f}<\bar{\Omega}^{f}$, the characteristic equation associated with the linearized counterpart of system (15) has $n^{-} \geq 1$ if $\rho^{\prime}\left(\pi_{*}\right) \in(0,1)$, and $n^{-}=0$ if $\rho^{\prime}\left(\pi_{*}\right)>1$.

Proof. See Appendix.

Lemma 5 implies that local indeterminacy of active monetary policies with a forward-looking feedback rule are ruled out by choosing a not too long forecasting horizon. This result can also be interpreted by continuity. Indeed, for $\Omega^{f}=0$, one gets a current-looking rule. Conversely, the case of an infinite horizon (which was analyzed by Benhabib et al. 2001) our sufficient condition is not satisfied and one may find multiple equilibria. This case is studied in the next Lemma.

Lemma 6. Let $\hat{\beta}^{f} \equiv \bar{\beta}^{f}\left[\frac{1}{\rho_{*}^{\prime}\left(\pi_{*}\right)}-\left(1-\chi^{f}\right)\right]$ and assume that $\Omega^{f} \rightarrow+\infty$. For $\rho^{\prime}\left(\pi_{*}\right) \in(0,1)$, the characteristic equation has $n^{-}=1$. For $\rho^{\prime}\left(\pi_{*}\right)>1$, the characteristic equation has $n^{-}=2$ if $\beta^{f}<\hat{\beta}^{f}$, and $n^{-}=0$ if $\beta^{f}>\hat{\beta}^{f}$. Proof. See Appendix.

To eliminate local indeterminacy, Lemma 6 suggests that the Central 
Bank should follow a very active (i.e. choose a large $\rho_{*}^{\prime}\left(\pi_{*}\right)$ ) monetary policy or a large $\beta^{f}$ if $\Omega^{f}$ is infinite. With Lemmas 5 and 6 , we conclude that reducing the forecasting horizon can be an alternative to an aggressive monetary policy. This limit for the degree of forward-lookingness is similar to some results that we have seen in discrete-time models with sticky prices by Batini and Nelson (2001), Batini and Pearlman (2002), Batini et al. (2004) and Batini et al. (2006). The main message we can take from this is that feedback should be at a horizon less than where equilibrium is reached.

\section{Conclusion}

In this article, we used recent stability theorems for functional differential equations in order to analyze the local dynamics of a flexible price and moneyin-the-utility-function model with backward- or forward-looking interest rate rules. We showed that the horizon over which the inflation rates are selected may play an important role in the determinacy of the equilibrium. With backward-looking feedback rules, an active policy leads to short-run fluctuations of nominal interest rates if the horizon is bounded. Those fluctuations vanish if the horizon is infinite. With forward-looking feedback-rules, an active policy leads to determinacy provided that the forecasting horizon is not too long.

As an extension of this work, it could be interesting to turn to normative considerations and define the optimal rule that allows the Central Bank to achieve its objectives. 


\section{Proofs}

Proof of Lemma 1. The system composed by equations (6), (7) and (9) rewrites as follows:

$$
\left\{\begin{array}{l}
R^{\prime}(t)=\Lambda(R(t))\left[R(t)-r-\frac{\rho^{-1}(R(t))-\chi^{b} \pi^{b}(t)-\chi^{f} \pi^{f}(t)}{1-\chi^{b}-\chi^{f}}\right] \\
\pi^{b}(t)=\frac{\int_{t-\Omega^{b}}^{t} e^{\beta^{b}(u-t)}\left[\rho^{-1}(R(u))-\chi^{b} \pi^{b}(u)-\chi^{f} \pi^{f}(u)\right] d u}{\left(1-\chi^{b}-\chi^{f}\right) \int_{-\Omega^{b}}^{0} e^{\beta^{b} u} d u} \\
\pi^{f}(t)=\frac{\int_{t}^{t+\Omega^{f}} e^{-\beta^{f}(u-t)}\left[\rho^{-1}(R(u))-\chi^{b} \pi^{b}(u)-\chi^{f} \pi^{f}(u)\right] d u}{\left(1-\chi^{b}-\chi^{f}\right) \int_{0}^{\Omega^{f}} e^{-\beta^{f} u} d u}
\end{array}\right.
$$

A Taylor approximation on the neighborhood of the steady-state transforms the previous system into:

$$
\left\{\begin{aligned}
R^{\prime}(t)= & \Lambda_{*}\left[\left(1-\frac{1}{\rho_{*}^{\prime}\left(1-\chi^{b}-\chi^{f}\right)}\right) R(t)+\frac{\chi^{b} \pi^{b}(t)+\chi^{f} \pi^{f}(t)}{1-\chi^{b}-\chi^{f}}\right] \\
& +\mathcal{M}\left(R(t), \pi^{b}(t), \pi^{f}(t)\right), \\
\pi^{b}(t)= & \frac{\int_{t-\Omega^{b}}^{t} e^{\beta^{b}(u-t)}\left[\frac{R(u)}{\rho_{*}^{\prime}}-\chi^{b} \pi^{b}(u)-\chi^{f} \pi^{f}(u)\right] d u}{\left(1-\chi^{b}-\chi^{f}\right) \int_{-\Omega^{b}}^{0} \beta^{\beta^{b} u} d u}+\frac{\int_{t-\Omega^{b}}^{t}{\beta^{\beta^{b}}(u-t)}^{f}(R(u)) d u}{\left(1-\chi^{b}-\chi^{f}\right) \int_{-\Omega^{b}}^{0} e^{\beta^{b} u} d u} \\
\pi^{f}(t)= & \frac{\int_{t}^{t+\Omega^{f}} e^{-\beta^{f}(u-t)}\left[\frac{R(u)}{\rho_{*}^{\prime}}-\chi^{b} \pi^{b}(u)-\chi^{f} \pi^{f}(u)\right] d u}{\left(1-\chi^{b}-\chi^{f}\right) \int_{0}^{\Omega^{f}} e^{-\beta^{f} u} d u}+\frac{\int_{t}^{t+\Omega^{f}} e^{-\beta^{f}}(u-t) \mathcal{N}(R(u)) d u}{\left(1-\chi^{b}-\chi^{f}\right) \int_{0}^{\Omega^{f}} e^{-\beta^{f} u} d u},
\end{aligned}\right.
$$

where $\Lambda_{*} \equiv \Lambda\left(R_{*}\right)$, and $\rho_{*}^{\prime} \equiv \rho^{\prime}\left(\pi_{*}\right)$, and where the nonlinearities write:

$$
\begin{aligned}
\mathcal{M}\left(R, \pi^{b}, \pi^{f}\right)= & {\left[\Lambda\left(R+R_{*}\right)-\Lambda\left(R_{*}\right)\right]\left[R-\frac{\frac{R}{\rho_{*}^{\prime}}-\chi^{b} \pi^{b}-\chi^{f} \pi^{f}}{1-\chi^{b}-\chi^{f}}\right] } \\
& -\frac{\Lambda\left(R+R_{*}\right) \mathcal{N}(R)}{1-\chi^{b}-\chi^{f}}, \\
\mathcal{N}(R)= & \rho^{-1}\left(R_{*}+R\right)-\rho^{-1}\left(R_{*}\right)-\frac{R}{\rho_{*}^{\prime}} .
\end{aligned}
$$

D'Albis et al. (2012) provide a linearization theorem for hyperbolic systems of differential-algebraic equations. Some conditions are standard and obviously satisfied for system (16). More importantly, both the linear and the non linear parts of the algebraic equations should reduce to functional differential 
equations when differentiated a finite number of times, which can easily be checked here by differentiating once with respect to time. Third, the non linear part and its first derivatives with respect to $\left(R, \pi^{b}, \pi^{f}\right)$ should vanish for $\left(R, \pi^{b}, \pi^{f}\right)=(0,0,0)$, which is also satisfied.

Proof of Lemma 2. The linearized counterpart of system (13) is obtained by substituting $\chi^{f}=0$ in the linear parts of the first two equations of system (17). We obtain:

$$
\left\{\begin{array}{l}
R^{\prime}(t)=\Lambda_{*}\left[\left(1-\frac{1}{\rho_{*}^{\prime}\left(1-\chi^{b}\right)}\right) R(t)+\frac{\chi^{b}}{1-\chi^{b}} \pi^{b}(t)\right], \\
\pi^{b}(t)=\kappa^{b} \int_{t-\Omega^{b}}^{t} e^{\beta^{b}(u-t)}\left[\frac{R(u)}{\rho_{*}^{\prime}}-\chi^{b} \pi^{b}(u)\right] d u,
\end{array}\right.
$$

where we recall that $\Lambda_{*} \equiv \Lambda\left(R_{*}\right)$, and $\rho_{*}^{\prime} \equiv \rho^{\prime}\left(\pi_{*}\right)$ and where we introduce $\kappa^{b} \equiv 1 /\left[\left(1-\chi^{b}\right) \int_{-\Omega^{b}}^{0} e^{\beta^{b} u} d u\right]$. The characteristic function of this system, denoted $\delta(z)$, is defined such that $\delta(z)=\operatorname{det}(\mathcal{I}(z))$ where:

$$
\mathcal{I}(z)=\left[\begin{array}{cc}
z-\Lambda_{*}\left(1-\frac{1}{\rho_{*}^{\prime}\left(1-\chi^{b}\right)}\right) & -\Lambda_{*} \frac{\chi^{b}}{1-\chi^{b}} \\
-\frac{\kappa^{b}}{\rho_{*}^{\prime}} \int_{-\Omega^{b}}^{0} e^{\left(\beta^{b}+z\right) \sigma} d \sigma & 1+\chi^{b} \kappa^{b} \int_{-\Omega^{b}}^{0} e^{\left(\beta^{b}+z\right) \sigma} d \sigma
\end{array}\right]
$$

Thus:

$$
\delta(z)=\left(z-\Lambda_{*}\right)\left(\chi^{b} \kappa^{b} \int_{-\Omega^{b}}^{0} e^{\left(\beta^{b}+z\right) \sigma} d \sigma+1\right)+\frac{\Lambda_{*}}{\rho_{*}^{\prime}\left(1-\chi^{b}\right)} .
$$

To prove the lemma, we proceed in two steps. $1 /$ we show there exists a unique positive real root if $\rho_{*}^{\prime}>1$ and that there is no positive real root if $\rho_{*}^{\prime} \in(0,1) .2 /$ we show there is no complex root with positive real parts.

$1 /$ Real roots of $\delta(z)=0$. Observe first that if $z-\Lambda_{*} \geq 0$, one has $\delta(z)>0$ and that if $z-\Lambda_{*}<0$, one has $\delta^{\prime}(z)>0$. Moreover, for $z \in$ $\left(0, \Lambda_{*}\right)$, one has $\delta(0)<\delta(z)<\delta\left(\Lambda_{*}\right)$ with $\delta(0)=\Lambda_{*}\left(1-\rho_{*}^{\prime}\right) / \rho_{*}^{\prime}\left(1-\chi^{b}\right)$ and $\delta\left(\Lambda_{*}\right)=\Lambda_{*} / \rho_{*}^{\prime}\left(1-\chi^{b}\right)$. Hence, if $1-\rho_{*}^{\prime}<0$, there exists a real root 
$z \in\left(0, \Lambda_{*}\right)$ such that $\delta(z)=0$ and if $1-\rho_{*}^{\prime}>0$, there is no real root $z \in\left(0, \Lambda_{*}\right)$ such that $\delta(z)=0$.

2/ Complex roots of $\delta(z)=0$. Let us denote the complex roots by $z=$ $p+i q$. We first prove that there are no complex roots with positive real part that satisfy $p>\Lambda_{*}$ by showing that $|\delta(z)|>0$. For $p>\Lambda_{*}$, one has:

$$
|\delta(z)|>\left|\frac{\Lambda_{*}}{\rho_{*}^{\prime}\left(1-\chi^{b}\right)}-\right|\left(\Lambda_{*}-z\right)||\left(\chi^{b} \kappa^{b} \int_{-\Omega^{b}}^{0} e^{\left(\beta^{b}+z\right) \sigma} d \sigma+1\right)|| .
$$

Then, it is sufficient to observe that the right-hand-side of the above inequality is greater than $\delta(p)>0$ to conclude. Let us now consider the roots whose real parts belong to $\left(0, \Lambda_{*}\right)$. We are going to show that in this case: $\operatorname{Im}(\delta(z))>q>0$. One has:

$$
\begin{aligned}
\operatorname{Im}(\delta(z))= & q\left[\chi^{b} \kappa^{b} \int_{-\Omega^{b}}^{0} e^{\left(\beta^{b}+p\right) \sigma} \cos (q \sigma) d \sigma+1\right] \\
& -\left(p-\Lambda_{*}\right) \chi^{b} \kappa^{b} \int_{0}^{-\Omega^{b}} e^{\left(\beta^{b}+p\right) \sigma} \sin (q \sigma) d \sigma .
\end{aligned}
$$

Thus:

$$
\operatorname{Im}(\delta(z))>q\left[\chi^{b} \kappa^{b} \int_{-\Omega^{b}}^{0} e^{\left(\beta^{b}+p\right) \sigma}[q \cos (q \sigma)+p \sin (q \sigma)] d \sigma+1\right] .
$$

Since:

$$
q \cos (q \sigma)+p \sin (q \sigma)=\left[\left(p^{2}+q^{2}\right) \int_{\sigma}^{0} e^{p u} \sin (q u) d u-q\right] e^{-p \sigma},
$$

one has:

$$
\operatorname{Im}(\delta(z))>q\left[\chi^{b} \kappa^{b} \int_{-\Omega^{b}}^{0} e^{\beta^{b} \sigma}\left[\left(p^{2}+q^{2}\right) \int_{\sigma}^{0} e^{p u} \sin (q u) d u-q\right] d \sigma+1\right] .
$$

Using the fact that $\int_{0}^{\sigma} e^{\left(\beta^{b}+p\right) u} \sin (q u) d u>0$ for $\sigma<0$, suffices to complete the proof. 
Proof of Lemma 3. When $\Omega^{b} \rightarrow+\infty$, the characteristic function (18) becomes

$$
\delta(z)=z^{2}+z\left[\frac{\beta^{b}}{\left(1-\chi^{b}\right)}+\Lambda_{*}\left(\frac{1-\rho_{*}^{\prime}\left(1-\chi^{b}\right)}{\rho_{*}^{\prime}\left(1-\chi^{b}\right)}\right)\right]+\frac{\Lambda_{*} \beta^{b}}{\left(1-\chi^{b}\right)}\left(\frac{1-\rho_{*}^{\prime}}{\rho_{*}^{\prime}}\right),
$$

for all $z>-\beta^{b}$. One has:

$$
\begin{aligned}
\delta\left(-\beta^{b}\right) & =-\frac{\chi^{b} \beta^{b}}{\left(1-\chi^{b}\right)}\left(\beta^{b}+\Lambda_{*}\right)<0 \\
\delta(0) & =\frac{\Lambda_{*} \beta^{b}}{\left(1-\chi^{b}\right)}\left(\frac{1-\rho_{*}^{\prime}}{\rho_{*}^{\prime}}\right)
\end{aligned}
$$

Hence, there is one real root that belongs to $\left(-\beta^{b}, 0\right)$ if $\rho_{*}^{\prime}<1$ and that is positive if $\rho_{*}^{\prime}>1$. The other real root is lower than $-\beta^{b}$, but the projection on the eigenvector related to this latter root is zero according to the definition of $\pi^{b}\left(0^{+}\right)$.

Proof of Lemma 4. The linearized counterpart of system (13) is obtained by substituting $\chi^{b}=0$ in the linear parts of the first and the third equations of system (17). We obtain:

$$
\left\{\begin{array}{l}
R^{\prime}(t)=\Lambda_{*}\left[\left(1-\frac{1}{\rho_{*}^{\prime}\left(1-\chi^{f}\right)}\right) R(t)+\frac{\chi^{f}}{1-\chi^{f}} \pi^{f}(t)\right] \\
\pi^{f}(t)=\kappa^{f} \int_{t}^{t+\Omega^{f}} e^{-\beta^{f}(u-t)}\left[\frac{R(u)}{\rho_{*}^{\prime}}-\chi^{f} \pi^{f}(u)\right] d u
\end{array}\right.
$$

where we recall that $\Lambda_{*} \equiv \Lambda\left(R_{*}\right)$, and $\rho_{*}^{\prime} \equiv \rho^{\prime}\left(\pi_{*}\right)$ and where we introduce $\kappa^{f} \equiv 1 /\left[\left(1-\chi^{f}\right) \int_{0}^{\Omega^{f}} e^{-\beta^{f} u} d u\right]$. The characteristic function of this system, denoted $\delta(z)$, is defined such that $\delta(z)=\operatorname{det}(\mathcal{I}(z))$ where:

$$
\mathcal{I}(z)=\left(\begin{array}{cc}
z-\Lambda_{*}\left(1-\frac{1}{\rho_{*}^{\prime}\left(1-\chi^{f}\right)}\right) & -\Lambda_{*} \frac{\chi^{f}}{1-\chi^{f}} \\
-\frac{\kappa^{f}}{\rho_{*}^{\prime}} \int_{0}^{\Omega^{f}} e^{\left(z-\beta^{f}\right) \sigma} d \sigma & 1+\chi^{f} \kappa^{f} \int_{0}^{\Omega^{f}} e^{\left(z-\beta^{f}\right) \sigma} d \sigma
\end{array}\right)
$$

which gives:

$$
\delta(z)=\left(z-\Lambda_{*}\right)\left(\chi^{f} \kappa^{f} \int_{0}^{\Omega^{f}} e^{\left(z-\beta^{f}\right) \sigma} d \sigma+1\right)+\frac{\Lambda_{*}}{\rho_{*}^{\prime}\left(1-\chi^{f}\right)} .
$$


Let us define $\bar{\beta}^{f} \equiv \Lambda_{*}$. We restrict to the case $\beta^{f} \geq \bar{\beta}^{f}$ and proceed in two steps by showing: $1 /$ there exists one negative real root if $\rho_{*}^{\prime} \in(0,1)$ and no negative real root if $\rho_{*}^{\prime}>1 ; 2 /$ there are no complex roots with negative real parts.

$1 /$ Real roots of $\delta(z)=0$. Let us compute the derivative of $(19)$ :

$$
\delta^{\prime}(z)=1+\chi^{f} \kappa^{f} \Omega^{f} e^{\left(z-\beta^{f}\right) \Omega^{f}}+\left(\beta^{f}-\Lambda_{*}\right) \chi^{f} \kappa^{f} \int_{0}^{\Omega^{f}} \sigma e^{\left(z-\beta^{f}\right) \sigma} d \sigma .
$$

For $\beta^{f} \geq \bar{\beta}^{f}$, one has $\delta^{\prime}(z)>0$. Since $\lim _{z \rightarrow-\infty} \delta(z)=-\infty$ and $\delta(0)=$ $\frac{\Lambda_{*}\left(1-\rho_{*}^{\prime}\right)}{\rho_{*}^{\prime}\left(1-\chi^{f}\right)}$, we conclude that there exists a unique negative real root if $\rho_{*}^{\prime} \in$ $(0,1)$ and no negative real root if $\rho_{*}^{\prime}>1$.

2/ Complex roots of $\delta(z)=0$. Let us denote the complex roots by $z=p+i q$.

One has:

$$
|\delta(z)|>\left|\left(1-\chi^{f} \kappa^{f}\left(1+\frac{\beta^{f}-\Lambda_{*}}{z-\beta^{f}}\right)\left(1-e^{\left(z-\beta^{f}\right) \Omega^{f}}\right)\right)+\frac{\Lambda_{*}}{\rho_{*}^{\prime}\left(1-\chi^{f}\right)}\right| .
$$

We conclude that for $\beta^{f} \geq \bar{\beta}^{f}$, one has: $|\delta(z)|>|\delta(p)|>0$.

Proof of Lemma 5. We show that for $\Omega^{f}$ small enough there exists one negative real root if $\rho_{*}^{\prime} \in(0,1)$ and no root with negative real part if $\rho_{*}^{\prime}>1$. We proceed in three steps:

$1 /$ There is a unique real root to $\delta(z)=0$ that is negative if $\rho_{*}^{\prime} \in(0,1)$ and positive if $\rho_{*}^{\prime}>1$. For $\Omega^{f}$ small, $\delta^{\prime}(z)>0$ (where $\delta^{\prime}(z)$ is given by (20)). We conclude with $\lim _{z \rightarrow-\infty} \delta(z)=-\infty, \lim _{z \rightarrow+\infty} \delta(z)=+\infty$, and $\delta(0)=\frac{\Lambda_{*}\left(1-\rho_{*}^{\prime}\right)}{\rho_{*}^{\prime}\left(1-\chi^{f}\right)}$.

$2 /$ For $\rho_{*}^{\prime}>1$, the positive real root, denoted $z_{1}$, is smaller than $\Lambda_{*}$. For $\Omega^{f}=0$, the characteristic function (19) rewrites (by applying l'Hôpital's Rule):

$$
\delta(z)=\frac{1}{1-\chi^{f}}\left[z-\Lambda_{*}\left(1-\frac{1}{\rho_{*}^{\prime}}\right)\right]
$$


For $\Omega^{f}$ small, $z_{1}$ is close to $\Lambda_{*}\left(1-\frac{1}{\rho_{*}^{\prime}}\right)$ and is thus smaller than $\Lambda_{*}$ for $\rho_{*}^{\prime}>1$. $3 /$ For $\rho_{*}^{\prime}>1$, there is no complex root $z=p+i q$, with real part smaller than $z_{1}$. One has:

$$
\begin{aligned}
\operatorname{Re}(\delta(z))= & \operatorname{Re}\left(\left(z-z_{1}\right) \chi^{f} \kappa^{f} \int_{0}^{\Omega^{f}}\left(e^{\left(z-\beta^{f}\right) \sigma}-e^{\left(z_{1}-\beta^{f}\right) \sigma}\right) d \sigma\right) \\
& +\operatorname{Re}\left(\left(z_{1}-\Lambda_{*}\right) \chi^{f} \kappa^{f} \int_{0}^{\Omega^{f}} e^{\left(z-\beta^{f}\right) \sigma} d \sigma\right)+\operatorname{Re}\left(\left(z-z_{1}\right)\right)
\end{aligned}
$$

Using the fact that $z_{1}<\Lambda_{*}$, we conclude that, for $\Omega^{f}$ small, $\operatorname{Re}(\delta(z))<0$ for all $z<z_{1}$.

Proof of Lemma 6. For $\Omega^{f} \rightarrow+\infty$, the characteristic function (19) is defined for $z \in\left(-\infty, \beta^{f}\right)$ and writes:

$$
\delta(z)=-z^{2}+\frac{z}{\left(1-\chi^{f}\right)}\left[\beta^{f}-\Lambda_{*}\left[\frac{1}{\rho_{*}^{\prime}}-\left(1-\chi^{f}\right)\right]\right]+\left(\frac{1}{\rho_{*}^{\prime}}-1\right) \frac{\Lambda_{*} \beta^{f}}{\left(1-\chi^{f}\right)},
$$

Since $\lim _{z \rightarrow-\infty} \delta(z)=-\infty$ and $\delta(0)=\left(\frac{1-\rho_{*}^{\prime}}{\rho_{*}^{\prime}}\right) \frac{\Lambda_{*} \beta^{f}}{\left(1-\chi^{f}\right)}$ we conclude there is one negative real root if $\rho_{*}^{\prime} \in(0,1)$ and either zero or two roots with negative real parts if $\rho_{*}^{\prime}>1$. The condition that excludes roots with negative real parts is $\delta^{\prime}(0)>0$, or equivalently $\beta^{f}>\Lambda_{*}\left[\frac{1}{\rho_{*}^{\prime}}-\left(1-\chi^{f}\right)\right]$. 


\section{References}

[1] H. d'Albis, E. Augeraud-Véron and H. J. Hupkes (2012), Discontinuous Initial Value Problems for Functional Differential-Algebraic Equations of Mixed Type. Journal of Differential Equations 253, 1959-2024.

[2] H. d'Albis, E. Augeraud-Véron and H. J. Hupkes (2013), Multiple Solutions in Systems of Functional Differential Equations. CES working papers 2013.07, University of Paris 1

[3] C. Azariadis (1981), Self-fulfilling Prophecies. Journal of Economic Theory $25,380-396$.

[4] N. Batini, A. Justiniano, P. Levine, and J. Pearlman (2006), Robust Inflation-forecast-based Rules to Shield Against Indeterminacy, Journal of Economic Dynamics and Control 30, 1491-1526

[5] N. Batini, P. Levine, and J. Pearlman (2004), Indeterminacy with Inflation-forecast-based Rules in a Two-bloc Model, International Finance Discussion Papers 797, Board of Governors of the Federal Reserve System (U.S.)

[6] N. Batini and E. Nelson (2001), Optimal Horizons for Inflation Targeting, Journal of Economic Dynamics and Control 25, 891-910

[7] N. Batini and J. Pearlman (2002), Too Much Too Soon: Instability and Indeterminacy with Forward-Looking Rules, Discussion Papers 08, Monetary Policy Committee Unit, Bank of England

[8] J. Benhabib (2004), Interest Rate Policy in Continuous Time with Discrete Delays. Journal of Money, Credit, and Banking 36, 1-15

[9] J. Benhabib and R. E. A. Farmer (1999), Indeterminacy and Sunspots in Macroeconomics, In: J. B. Taylor and M. Woodford (Eds.), Handbook of Macroeconomics 1A, 387-448, Elsevier

[10] J. Benhabib, S. Schmitt-Grohé and M. Uribe (2001), Monetary Policy and Multiple Equilibria. American Economic Review 91, 167-186

[11] J. Benhabib, S. Schmitt-Grohé and M. Uribe (2002), Avoiding Liquidity Traps. Journal of Political Economy 110, 535-563.

[12] J. Benhabib, S. Schmitt-Grohé and M. Uribe (2003), Backward-Looking Interest Rate Rules, Interest-Rate Smoothing, and Macroeconomic Instability. Journal of Money, Credit, and Banking 35, 1379-1412

[13] B. Bernanke and M. Woodford (1997), Inflation Forecasts and Monetary Policy. Journal of Money, Credit and Banking 29, 653-684

[14] R. Boucekkine, D. de la Croix and O. Licandro (2004), Modelling Vintage Structures with DDEs: Principles and Applications, Mathematical Population Studies 11, 151-179 
[15] R. Boucekkine, G. Fabbri and P. Pintus (2012), On the optimal control of a linear neutral differential equation arising in economics, Optimal Control, Applications and Methods 33, 511-530

[16] C. T. Carlstrom and T. S. Fuerst (2000), Forward-Looking Versus Backward-Looking Taylor Rules. Working paper 00-09, Federal Reserve Bank of Cleveland.

[17] C. T. Carlstrom and T. S. Fuerst (2001), Timing and Real Inteterminacy in Monetary Models. Journal of Monetary Economics 47, 285-298

[18] C. T. Carlstrom and T. S. Fuerst (2003), Continuous versus Discretetime Modeling: Does it make a Difference? Mimeo

[19] C. T. Carlstrom and T. S. Fuerst (2005), Investment and Interest Rate Policy: A Discrete Time Analysis. Journal of Economic Theory 123, $4-20$

[20] R. Clarida, J. Galí and M. Gertler (1998), Monetary Policy Rules in Practice: Some International Evidence. European Economic Review 42, 1033-1067

[21] R. Clarida, J. Galí and M. Gertler (2000), Monetary Policy Rules and Macroeconomic Stability: Evidence and Some Theory. The Quarterly Journal of Economics 115, 147-180

[22] J. H. Cochrane (2011), Determinacy and Identification with Taylor Rules. Journal of Political Economy 119, 565-615

[23] B. Dupor (2001), Investment and Interest Rate Policy. Journal of Economic Theory $98,85-113$

[24] S. Eusepi (2005), Comparing Forecast-Based and Backward-Looking Taylor Rules: A "Global" Analysis. Federal Reserve Bank of New York Staff Reports, no 198.

[25] E. Leeper (1991), Equilibria under 'Active' and 'Passive' Monetary and Fiscal Policies. Journal of Monetary Economics 27, 129-147

[26] P. Levine, P. McAdam and J. Pearlman (2007), Inflation-Forcast6Based Rules and Indeterminacy: a Puzzle and a Resolution. International Journal of Central Banking 3, 77-110

[27] A. Orphanides (2001), Monetary Policy Rules Based on Real-Time Data. American Economic Review 91, 964-985

[28] T. J. Sargent and N. Wallace (1975), "Rational" Expectations, the Optimal Monetary Instrument, and the Optimal Money Supply Rule. Journal of Political Economy 83, 241-254

[29] J. B. Taylor (1993), Discretion Versus Policy Rules in Practice. CarnegieRochester Conference series on Public Policy 39, 195-214 
[30] M. Woodford (1999), Optimal Monetary Policy Inertia, NBER working paper no. 7261

[31] M. Woodford (2003), Interest and Prices: Fondations of a Theory of Monetary Policy. Princeton University Press, Princeton 